\title{
Les affaires du mort
}

The Things of the Deceased (Tamberma, North Togo)

\section{Myriam Smadja}

\section{OpenEdition}

\section{Journals}

Édition électronique

URL : http://journals.openedition.org/span/1230

DOI : $10.4000 /$ span. 1230

ISSN : 2268-1558

\section{Éditeur}

École pratique des hautes études. Sciences humaines

\section{Édition imprimée}

Date de publication : 1 décembre 1991

Pagination : $57-90$

ISSN : 0294-7080

\section{Référence électronique}

Myriam Smadja, «Les affaires du mort », Systèmes de pensée en Afrique noire [En ligne], 11 | 1991, mis en ligne le 14 novembre 2013, consulté le 01 mai 2019. URL : http://journals.openedition.org/ span/1230; DOI : 10.4000/span.1230 


\title{
LES AFFAIRES DU MORT
}

\author{
par \\ Myriam Smadja
}

Par le mot tinenti, les Batammariba ${ }^{3}$ entendent: "les choses inséparables d'une personne" ou "solidement attachées à son corps". Par exemple, les bracelets de fer que l'on fait porter à un enfant lorsque le "souffle" du mort "revenu" en lui à la naissance a été identifié par les devins. Tincnti par excellence, ces bracelets ne quitteront les poignets d'une personne qu'au moment où l'on se préparera à l'ensevelir. Formé sur le même radical que le verbe kennei ("accompagner") qui implique l'idée d'une intimité profonde entre deux personnes, le terme tinعnti désigne essentiellement les "affaires" ou "effets" $\mathbf{d}$ 'un ancien exhibés au portail de sa maison lors du rite des tibenti ("choses des tambours") ${ }^{4}$. Ce rituel marque l'ouverture d'une nouvelle tombe

\footnotetext{
' Aux confins du Bénin et du Togo, dans la plaine et les massifs de l'Atakora, vivent les Batanmariba (sing. Otanmari, "pétrisseur de terre humide"), célèbres pour la construction de leurs forteresses de banco. Chasseurs, agriculteurs, éleveurs, ils affirment être venus du nord. Une étude approfondie de leurs migrations a été menée par Paul Mercier (1968). Appartenant à ce qu'il est convenu d'appeler une société "sans état", les Batarmariba sont appelés Tamberma au Togo et Somba au Bénin. Toutes mes recherches ont été effectuées au Togo.

Tout ancien, home ou femme, ayant survécu à dix rituels initiatiques a droit à sa mort au rite tibenti ("choses des tambours"). Il s'agit des tambours que l'on fait résonner à difuani, rituel initiatique masculin marquant le passage à l'âge adulte, qui a lieu tous les quatre ans, et qui succède à dikunteri d'un même clan âgés de 18 à 22 ans. C'est donc vers l'âge de 60 ans qu'un ancien peut bénéficier d'un tibenti, qu'il soit marié, veuf ou célibataire, qu'il ait ou non des enfants. En ce dernier cas, ce sont les membres de la fratrie à laquelle appartenait le défunt qui prendront en charge la cérémonie. Quel que soit le sexe du défunt, la chronologie des rites funéraires est identique:
}

Systèmes de pensée en Afrique Noire, 11, 1991 
dont le défunt devient le propriétaire ou le gardien, comme de son vivant il était maître de sa takienta, sa maison forteresse. Sur son corps seront à l'avenir déposés les jeunes morts de sa famille. Les tincnti sont de deux sortes: les "effets" personnels du défunt, ukinenti (de uki "défunt" et de nenti "affaires") et les "affaires de mort", tikunenti (de ku "mort" et tinenti "affaires"). Parmi ces dernières, on distingue le simulacre du défunt (une petite branche de baobab), les instruments des fossoyeurs et les objets utilisés pour le traitement du cadavre d'une part, et, d'autre part, les instruments de musique et les parures réservées au rituel tibenti et aux deux levées de deuil. Ces derniers tikunenti (instruments et parures) appartiennent au clan du mort et ne sont utilisés que pour le tibenti des anciens de ce clan. Les "effets" du mort (ukinenti) sont les choses qui ne quittaient pas le corps de la personne de son vivant. Ils sont de trois sortes: supports de puissance masculine (arc et carquois) ou féminine (bâton de marche), prolongements du corps en tant qu'organisme vivant recouvert de peau" (sac, pipe, ongles, cheveux), et matérialisation du souffle - diyanni - d'un mort "revenu" dans une personne (bracelets de fer ou cuivre).

Un cadavre est couché nu dans la tombe, sans parure ni objet à proximité. Les "affaires", tant du mort que de mort, seront traitées à des moments différents du rituel. Certaines seront détruites, d'autres conservées. Le sort qui leur est réservé semble être fonction du type de liens qu'elles symbolisent: relation du défunt avec sa maison, son lignage, son clan, ou encore avec le "souffle" du mort revenu en lui au moment de sa conception. Certains objets seront brisés puis enfouis dans la terre lors de la première levée de deuil, bakunaa (de banaa "bière de mil" et ku "mort"), d'autres seront "jetés" au cimetière, à la seconde levée de deuil qui, selon le sexe

collecte des "affaires de mort", creusement de la tombe, interrogatoire du défunt pour déterminer la cause du décès, enterrement au cimetière, veille du simulacre du défunt durant la nuit à l'intérieur de la maison, rejet du simulacre au crépuscule. Quatre jours plus tard, le jour de la première levée de deuil, a lieu la fermeture de la tombe. Une deuxième levée de deuil est marquée par la construction d'un autel dans la maison du défunt.

${ }^{3}$ Corps et peau se disent du même mot: tikpanti. 
du défunt, a lieu six mois ou cinq à dix ans après le décès. D'autres encore, tels que l'arc ou le bâton de marche, seront éliminés clandestinement bien après la seconde levée de deuil. On doit alors convenir que la manipulation des tincnti rythme, parfois de manière syncopée, la transmutation qu'accomplit le rituel sur la personne du défunt. De mort ordinaire, ou de mort-cadavre, celui-ci deviendra, au terme des levées de deuil, ce mort dont le souffle (diyanni) pourra déclencher le processus d'une nouvelle vie humaine dans le ventre d'une femme. Mais cette transmutation ne se fait pas sans à-coups, ni, si l'on peut dire, sans repentirs et retours en arrière: on dirait que l'on veut tantôt chasser le défunt de sa maison, tantôt l'inciter à y retourner - en un lieu soigneusement circonscrit cependant. Chacune des étapes se solde par la destruction ou la conservation de certains tinenti et par le réaménagement des relations que, désormais, les survivants entretiendront avec le défunt.

Dans la première partie de cet article, après avoir présenté les différentes catégories de tinєnti, j'essaierai de mettre en évidence les enjeux de la réunion de ce type d'objets en prenant pour exemple la collecte rituelle des "affaires de mort" au cours des funérailles d'un homme. Dans une seconde partie, je tenterai une première interprétation de ce qui se joue dans la destruction, après l'enterrement, des effets personnels d'un défunt. Je m'appuierai, cette fois, sur un rite observé lors du tibenti d'une femme. Précisons toutefois dès maintenant que, quel que soit le sexe du défunt, collecte et destruction des tinenti s'accomplissent en effet de façon identique.

\section{LES AFFAIRES DE MORT}

Le jour succédant à l'inhumation d'un ancien est marqué par la joie exubérante des jeunes. A quelque distance de la maison en deuil, les jeunes venant de villages rivaux forment un cercle où les improvisateurs les plus réputés s'affrontent en joutes de chant. Dans la pièce du bas de la maison, les femmes âgées dansent depuis la veille auprès du simulacre du corps du défunt, "pour la satisfaction du mort". Ce 
simulacre, fait d'une branche de baobab à quatre tronçons, longue de $25 \mathrm{~cm}$ environ, évoque un corps humain en quelque sorte androgyne: orné des parures des initiés, il comporte tout à la fois celles de difuani (rituel initiatique masculin) et celles de dikunteri (rituel initiatique féminin). Pendant ce temps, au cimetière, oncles utérins et frères du défunt consomment une boule de mil sans sauce ni condiments. Ils reviennent au crépuscule, en une longue file. Dès que l'on entend les sons de leur tambour, au loin, les réjouissances prennent fin à la maison du mort. Chacun sait que les hommes reviennent pour prendre le simulacre afin d'aller le "jeter dans la brousse" (en fait, au cimetière). Quand la procession est en vue, une femme, coiffée du casque à cornes de gazelle des initiées et armée de leur pique, s'élance hors de la maison. Dans un va-et-vient rapide, elle feint de provoquer les deux hommes` en tête de file qui portent à l'épaule les tinenti du mort. Que la guerrière menace les tinenti ou bien ceux qui les exhibent, qu'elle leur interdise le seuil de la maison ou au contraire les incite à le franchir - sa lance pointée vers le portail semble les guider - est une énigme loin d'être résolue. Elle ne sera pas abordée ici. Bornons-nous à considérer le plan de la séquence où, dans une marche lente et comme inéluctable, la procession se dirige vers la maison. C'est le seul moment du rituel où les "affaires" du mort sont montrées ensemble. L'homme qui marche en tête porte les tikuncnti ("affaires de la mort") liées à la tombe et au traitement du cadavre; derrière lui, un autre porte les ukinenti ("effets personnels du mort"). Dès que le simulacre aura quitté la maison, ces tinenti seront suspendus à la façade, près du portail. Jusqu'à la levée de deuil, ils recomposeront une première image du défunt, et resteront à la limite du dehors et du dedans de la takienta: à la lisière de deux mondes.

4 Leur fonction est également de mettre en terre le cadavre dans la position appropriée: une femme sur le côté gauche, les pieds au nord, un home sur le côté droit, les pieds au sud. 


\section{L'enjeu des tikunenti}

Les tikungnti ("affaires de la mort") sont de deux sortes. Celles que l'on exhibe au portail, et qui intéressent la tombe et le cadavre, sont des objets quotidiens, d'une grande banalité. lls présentent pourtant une particularité essentielle: utilisés une seule fois, ils doivent avoir l'aspect du neuf le jour du creusement de la tombe. Par contre, les instruments de musique que l'on fait résonner pendant la nuit de veille du simulacre et les parures qui ornent celui-ci font partie du patrimoine du clan". Ces objets appartiennent aussi bien aux "choses du tambour" qu'aux cérémonies initiatiques organisées à l'intérieur du clan. Leur histoire se confond avec celle de la "vieille takienta" de clan où ils sont gardés. Ils se composent des instruments de musique: trompes, sonnailles et les tambours qui donnent leur nom au rituel, et des parures d'initiés qui associent le casque à cornes de dikunteri et les colliers de cauris portés à la fois par les garçons et les filles.

Les tikunenti de la tombe et du cadavre sont des objets faits de matériaux périssables, à l'exception de la houe du cimetière. La composition de ces "affaires de la mort" est la suivante :

1) les instruments des fossoyeurs :

- un panier sans anse, dans lequel les femmes ont coutume de transporter graines ou haricots. Au cimetière, les bords du panier limiteront le tracé de la "bouche" de la tombe.

- une calebasse semblable à la calebasse individuelle où l'on prend son repas. Elle permettra de déblayer la terre de la fosse.

- une houe à fer étroit, nommée tapienta, que les hommes emploient pour abattre des arbres. A la base d'une cheminée étroite, le fossoyeur aménagera avec cet instrument la "chambre" du défunt.

- un pieu à pointe métallique servant à déterrer les ignames. C'est avec ce pieu qu'on entame la terre et que l'on creuse la cheminée. Quand un Otammari parle de la kuya, la "hache du cimetière", il fait allusion aux deux instruments, houe et pieu confondus.

\footnotetext{
" Le village de Kuwadaku ò̀ j'ai poursuivi mes recherches comprenait deux clans subdivisés en sous-clans (que je qualifie de complémentaires).
} 
- une poterie ronde ou ovale selon le sexe du défunt. La première réservée aux femmes sert à cuire la pâte de mil. Dans la seconde, on préserve à l'ombre la bière de mil. Cette poterie sera fixée sur le tertre funéraire le jour de la levée de deuil.

2) les objets servant au traitement du cadavre et du simulacre:

- un bol de beurre de karité. La deuilleuse en enduira le corps après l'avoir lavé et le simulacre.

- un cordon de fibres noires et rouges, identique au collier de hanches porté par les initiées à la fin du dikunteri.

- une botte de paille. Elle servira à tresser la natte du simulacre.

- quatre feuilles d'un arbre de karité, provenant d'un village où "un homme infortuné est mort sans qu'on lui creuse de tombe". Elles seront utilisées à la levée de deuil.

L'originalité de ces tikunenti (instruments des fossoyeurs et objets des deuilleuses) tient essentiellement à la manière dont on les réunit et à la spécifité sociale et sexuelle qui leur est dévolue. En effet, les Batammariba distinguent les tikunenti féminins des tikunenti masculins, quel que soit par ailleurs le sexe du défunt. Ainsi, la kuya métallique, instrument lié aux activités spécifiques des hommes (défrichage, creusement des sillons) est attribut masculin; c'est du reste le seul tikunenti masculin. Il revient au fils benjamin, futur héritier de la takienta, de se procurer l'instrument avec lequel on creusera la tombe de son père. Il commence à s'en occuper dès que ce dernier approche de l'âge donnant droit au tibenti. La hache, cachée dans la maison, ne doit jamais tomber sous le regard du père. La lame doit conserver l'éclat du neuf. Si elle vient à ternir, le fils en commande une autre chez le forgeron. Quant aux tikunenti que sont le panier, la calebasse, la paille ..., ils représentent la femme dans ses activités domestiques. Ils sont sous la responsabilité de la n'kua, ou fille aînée, qui fait preuve de la même discrétion que son frère pour les réunir. Eux aussi doivent présenter l'aspect du neuf, malaisé à conserver en raison de la nature végétale ou argileuse donc périssable - de leur matériau.

Les fossoyeurs ne peuvent pas commencer à creuser la tombe avant que le responsable du tibenti ne leur ait présenté les instruments. Et celui-ci ne les apporte que lorsque tous les tikunenti sont 
réunis. Les objets cérémoniels (instruments de musique et parures d'initié) sont immédiatement disponibles; il suffit de se rendre à la vieille takienta de clan". Quant à la kuya, tenue en réserve par le benjamin dans la takienta paternelle, il suffit de la sortir de sa cachette. Futur héritier de la maison, un benjamin doit éviter de quitter le village et, jusqu'à sa propre mort, être constamment en mesure de défendre sa takienta. Mais la réunion des tikunenti féminins donne lieu à des controverses aussi imprévisibles que peut l'être le caractère des femmes (si l'on en croit les hommes). Se procurer une calebasse, un panier, voire une poterie, semble à première vue fort aisé, même si ces objets doivent présenter l'aspect du neuf: chaque maison en est abondamment pourvue. Pourtant le creusement d'une tombe est parfois précédé d'un laps de temps plus ou moins long pendant lequel règne une incertitude qui frise l'angoisse: les tikunenti seront-ils réunis à temps ? Les fossoyeurs doivent avoir terminé avant le crépuscule et leur travail demande six à huit heures d'efforts intenses.

Ainsi, le soleil est déjà haut, et le problème de la réunion des tikunenti féminins loin d'être résolu, ce jour de juin 1982 où les fossoyeurs attendent qu'on leur apporte les "affaires" de la tombe: calebasse, panier, kuya. Après consultation divinatoire, l'un des clans du village a décidé d'organiser le tibenti d'un homme mort quelques mois plus tôt, vers l'âge de cinquante cinq ans. Sans descendance, surnommé "le célibataire" (abandonné par son épouse, il avait été voué à l'ironie de son entourage), il a été enterré "comme un jeune" dans la tombe d'un ancien". Selon le dire des devins, "il retient la pluie" pour se venger de l'affront. Par sa faute, les semailles ne peuvent débuter.

On a couché son simulacre sous le grenier masculin". Quatre à cinq femmes entourent la branche, agitant le chasse-mouches céré-

\footnotetext{
'A défaut, dans l'une ou l'autre takienta du clan où, par cormodité, sont gardés ces objets.

'Bien que mort légèrement avant $l$ 'âge requis, cet horme aurait pu bénéficier d'un tibenti. Mais sans enfant, peu estimé à cause de son manque de générosité, vivant dans la solitude, il avait été rapidement enterré et personne n'avait insisté pour faire la cérémonie.

${ }^{8}$ La takienta - orientée à l'ouest - a deux greniers, situés sur la terrasse: au sud, le grenier nasculin, au nord le grenier féminin.
} 
moniel, tout comme si elles veillaient le cadavre d'un père avant l'enterrement. Mais les chants de deuil alternent avec des remarques acrimonieuses. Assises de part et d'autre du simulacre, deux femmes âgées d'une quarantaine d'années déplorent le retard de la $n^{\prime} k u a$, la fille aînée. Le jour du décès, il échoit à cette femme d'apporter les tikunenti à la maison du deuil afin de les remettre au responsable de la cérémonie. Les deuilleuses trompent leur attente en passant en revue les insuffisances de la retardataire: non seulement cette $n ' k u a$ est "coléreuse, peu serviable et facilement irritée", mais "elle ne s'est jamais souciée des tikungnti". Pire: "elle refuse de s'en occuper, malgré la tante paternelle qui l'a désignée !". Quand la n'kua fait son apparition sur la terrasse, on l'accueille avec des sarcasmes: "elle n'apporte que la calebasse et la corde du brancard ... et le panier, où est-il ?... et la paille pour tresser la natte ?". D'autres femmes - des soeurs - arrivent à leur tour. La discussion s'envenime. On prend à parti la n'kua, indifférente aux remous. On lui reproche de n'avoir pas fait appel, en temps utile, à l'une ou l'autre de ses soeurs: "laquelle aurait refusé de l'aider ?". La situation se complique, personne ne veut aller au pied de la montagne pour couper la paille: "c'est trop loin !". Finalement, les tikunenti féminins sont réunis au milieu du jour. Les fossoyeurs peuvent commencer leur travail.

La mauvaise volonté des deuilleuses, compréhensible dans ce contexte d'enterrement simulé d'un homme dont on n'apprécie guère la vengeance d'outre-tombe, est une caricature à peine chargée des différends que suscite souvent la collecte des tikunenti féminins. Ces échanges d'amabilités reçoivent un éclairage nouveau lorsque l'on connaît la place tenue par les responsables de ces "affaires" à l'intérieur de la petite et de la grande ou vieille takienta.

Une takienta est une maison à étage regroupant une famille restreinte. Désignée sous le nom de "maison de l'épouse", elle appartient au mari. Seul un benjamin" hérite de la takienta paternelle. Les autres frères construisent leur propre maison - "petite takienta" lorsqu'ils se marient. Un groupe de frères est lié par la propriété commune d'un troupeau de boeufs sacrificiels. Cette fratrie porte le

\footnotetext{
'A défaut de fils, une fille peut hériter de la maison.
} 
nom de kunalakua ("tuer le boeuf ensemble"). Lors de toute cérémonie d'importance, les frères sacrifient un boeuf sur les autels d'ancêtre de la takienta paternelle ou vieille takienta. Comme les fratries ont tendance à se scinder facilement ${ }^{10}$, la vieille takienta appartient souvent à un ancien dans l'orbite duquel un petit nombre de frères accepte de vivre. Un clan, fawafa ("serpent") regroupe plusieurs kunalakua. Un village compte généralement deux, quatre ou six clans. Un clan ressoude les liens des différentes vieilles takienta de fratries au travers des grandes cérémonies d'initiation qui ont lieu tous les quatre ans. Les jeunes garçons de même fawafa sont alors initiés ensemble à la vieille takienta clanique, distincte des vieilles takienta de fratries.

La collecte des tikungnti intéresse essentiellement la fratrie du défunt. Deux femmes tiennent les rôles principaux: la soeur aînée (ou présumée telle) du défunt et la fille aînée de ce dernier. L'une des fonctions d'une tante paternelle est d'inciter une fille aînée, $n^{\prime} k u a$, à se procurer les différents tikunenti de son père quand celui-ci approche de l'âge donnant droit au rituel. Cette $n^{\prime} k u a$ peut être une fille réelle ou classificatoire (de même kunalakua). Elle peut aussi être une cadette, tout dépend du choix fait par la tante paternelle qui jette son dévolu sur la fille lui paraissant réunir les qualités nécessaires à cette tâche: constance de caractère (une épouse volage est peu souhaitable), proximité du lieu de résidence. Une n'kua digne de ce nom doit veiller à ce que chacune de ses soeurs soit responsable d'un tikunenti. Elle doit régulièrement contrôler l'intégrité de ces objets qui, ne l'oublions pas, doivent présenter l'aspect du neuf le jour de l'enterrement. Gardées dans la maison d'un frère ou dans la maison paternelle, les "affaires" doivent pouvoir être aisément réunies le jour du décès.

Ceci est la règle. Mais les obstacles à la collecte des tikunenti tiennent principalement au fait qu'aussi bien la $n^{\prime} k u a$ que ses soeurs ont depuis longtemps quitté la maison paternelle quand il leur faut songer à réunir les "affaires". Dans l'exemple de la collecte laborieuse des tikunєnti du "célibataire", les deuilleuses, si sévères dans leurs

\footnotetext{
${ }^{10}$ La raison invoquée est celle de la mésentente entre les frères.
} 
remontrances, semblaient oublier que toute fille mariée et mère de famille connaît, à un moment ou à un autre, des vicissitudes conjugales. Certaines se font "enlever" par un nouvel époux, parfois à plusieurs reprises, en particulier lorsqu'elles sont stériles et espèrent ainsi devenir mères. Leur famille d'origine n'est pas toujours mise au fait de leur changement de domicile.

L'ajde que la n'kua est en droit d'attendre de ses soeurs est donc compromise, quel que soit le sérieux avec lequel elle envisage ses fonctions. A la limite, la benjamine mariée tardivement et considérée comme la plus proche affectivement de son père serait la mieux désignée pour remplir ce rôle, mais précisément, cette éventualité n'entre pas dans la logique du rassemblement des tikungnti. L'aînée, ou présumée telle, doit être seule responsable. Les difficultés réelles ou simulées à réunir les "affaires" traduisent la vocation profonde de la collecte des tikunenti féminins: rassembler pour un court laps de temps, autour de la dépouille d'un père ( $d$ 'une mère' " ou d'une tante paternelle) la totalité des soeurs d'un kunalakua. En quelque sorte, gommer provisoirement leur état de femmes mariées: faire comme si les soeurs n'avaient jamais quitté ni la takienta paternelle ni le kunalakua. Au moment où l'on veille le corps exposé sous l'un des greniers de la terrasse, les gendres n'accompagnent pas leurs épouses, ils se rendent au cimetière du clan auquel appartenait le défunt pour prêter leur concours aux fossoyeurs'? Juste avant l'enterrement, la takienta, et plus largement la fratrie, reconstitue son intégralité et son unité, hors de la présence de l'étranger que reste un mari. Les deuilleuses qui, sous forme de remontrances, énumèrent les devoirs auxquels sont soumises les filles au cours de la collecte, rappellent, en fait, la place qu'occupe chacune d'elles à l'intérieur de la takienta familiale comme à l'intérieur de la fratrie. Rejouant sur la terrasse, auprès du cadavre allongé, la comédie de la jeunesse, les soeurs offrent l'image éphèmère du kunalakua tel

\footnotetext{
"Le déroulement du tibenti d'une mère est un peu plus complexe puisque les diverses cérémonies ont lieu successivement dans la takienta du mari et dans la takienta du père de la défunte. Une femme, quel que soit son âge, est toujours enterrée dans le cimetière du clan de son père.

12 De même, les époux creusent la tombe de leur belle-mère, mais cette fois dans le cimetière du clan paternel de celui-ci.
} 
qu'il se présentait avant leur départ de la maison paternelle - avant leur mariage. Surtout, elles donnent forme à un kunalakua "mythique" tel que le réalise au cimetière la configuration de la tombe. Chaque clan a son propre cimetière, où sont enterrés les frères et les soeurs de même kunalakua ${ }^{13}$. Une tombe, creusée pour un ancien, double et prolonge une vieille takienta de fratrie. Les jeunes morts adultes sont enterrés, selon leur sexe, dans la tombe d'un père ou d'une tante paternelle "propriétaire" de la sépulture. La recherche, puis la collecte des tikuncnti féminins traduit bien alors la volonté occulte de la vieille takienta de rétablir pour un temps très court le lien privilégié frère-soeur sur lequel se fonde l'unité du kunalakua.

\section{Le destin des tikunenti.}

$\mathrm{Au}$ soir du creusement de la tombe, on a peine à reconnaître dans le panier déchiré et la calebasse fendue et terreuse, les tikunenti flambant neufs du matin. La fragilité de leur matériau, l'aspect vétuste qui les caractérise une fois le corps mis en terre, reflètent le risque de aétérioration rapide auquel sont exposés les liens de kunalakua et finalement de takienta. En effet, une fois le corps inhumé, calebasse et panier de la tombe sont accrochés à une branche fourchue à laquelle ont été suspendus les crânes des boeufs donnés par les fils aux parents maternels du défunt, et sacrifiés chez ces derniers. Le tout - branche fourchue, crânes, calebasse et panier porte le nom de tiyoti ("saletés") du mort. Ces tiyoti sont exhibés au portail de la maison du défunt, à coté des "effets personnels", dès le lendemain de l'enterrement. Mais, alors que la plupart des "effets personnels" seront détruits à la première levée de deuil, bakunaa, quelques jours après le décès, les tiyoti seront détruits au cimetière à la seconde levée de deuil. Celle-ci a lieu six mois après le décès d'une femme, de un à deux ans après le décès d'un homme. "Les tiyoti d'une mère sont dangeureux, il faut s'en débarrasser au plus tôt"

\footnotetext{
${ }^{13}$ Un village composé de six clans peut avoir six cimetières. Parfois, les membres de deux clans sont enterrés dans le même cimetière. Leurs liens réciproques se reflètent dans l'existence d'une takienta cérémonielle commune où les jeunes des deux clans sont initiés ensemble.
} 
disent les Batammariba. Mais pourquoi attendre, au minimum, six mois pour procéder à cette destruction ? Pourquoi, surtout, une seconde levée de deuil ? Les réflexions de R. Hertz (1907) sur les rites de deuil suggèrent une réponse à cette question. Hertz remarque que les secondes funérailles en Indonésie correspondent au moment où le cadavre, entièrement décomposé, se réduit à l'ossature. Ce laps de temps qui sépare l'instant de l'inhumation de celui de ces cérémonies serait nécessaire, d'une part à la transmutation du mort en ancêtre, d'autre part au réaménagement des relations entre survivants et mort. Pour les Batammariba, il semblerait qu'il y ait correspondance entre l'état de dessèchement des crânes d'animaux six mois après l'enterrement, et celui qui, selon eux, caractériserait un corps réduit à l'état de squelette dans la tombe. C'est à ce moment que le souffle, diyanni, d'un mort serait en mesure de sortir de la tombe pour "revenir" dans un nouvel être humain. Détruire les "saletés" d'un mort à la seconde levée de deuil serait entériner publiquement, officialiser, la décomposition complète du cadavre rendant possible le "retour" du souffle d'un mort dans un vivant.

Le sort des tikunenti féminins tranche avec celui de la kuya (hache et pieu de la tombe), "affaire de mort" masculine, et des tikunenti cérémoniels (instruments de musique, parures d'initiés). La kuya est conservée: avec elle sera de nouveau ouverte la tombe qu'elle a permis une première fois de creuser. On y ensevelira les jeunes morts adultes de même kunalakua. Outil de construction de la demeure d'un défunt "propriétaire de sa sépulture", elle est gardée par le benjamin dans la takienta dont il hérite. Cette kuya, liée à une oeuvre de construction - la tombe - qui doit perdurer, confirme la pérennité du lien entre le maître d'une maison, le fils benjamin qui prend sa place et les autels d'ancêtres qui seront désormais sous la responsabilité de ce dernier: il veillera à leur régénération grâce à des sacrifices appropriés. Quant aux tikunenti cérémoniels, interchangeables $^{14}$, ils manifestent le lien indéfectible de tout membre d'un kunalakua avec la vieille takienta de clan où il a été initié dans sa jeunesse.

\footnotetext{
14 Ils sont indifférement utilisés pour tout tibenti d'ancien appartenant au clan. En particulier, les tambours sont aussi bien "choses" de difuani qu"affaires" de tinenti.
} 
En résumé, les tikunenti sont donc de deux sortes. Les uns, liés au corps-cadavre, ont une durée de vie équivalente au temps de décomposition d'un corps. La singularité de leur collecte, faite par les soeurs, témoigne à la fois de la mouvance des liens de kunalakua et d'une volonté occulte de maintenir une union dépendant essentiellement du lien frère-soeur. Les autres, liés à une vieille takienta - de clan et de kunalakua - au travers de la tombe et du cimetière, sont affaires de bâtisseurs, au sens que pourrait leur donner un Otammari, "pétrisseur de terre humide", et fondateur d'une takienta destinée à devenir, au cours des générations, "vieille takienta".

\section{LES EFFETS PERSONNELS DU MORT}

Bien avant que les tikunenti soient rassemblés à la maison de deuil, les effets personnels d'un mort, ukinenti, sont disposés auprès du corps lavé et oint de beurre de karitéts. Aucune difficulté à les réunir: le jour du décès, on se borne à retirer bracelets et parures corporelles et à rassembler les quelques objets sans lesquels un Otammari ne se déplace pas.

Ces effets sont, nous l'avons vu, de trois sortes: supports d'une puissance spécifiquement masculine ou féminine, prolongements du corps individualisé (ils en gardent la trace et l'odeur), lien unissant une personne au souffle de l'ancêtre revenu en elle.

Les effets d'un homme comprennent:

- l'arc et le carquois, signe de courage et de dignité masculine. L'arc est le support de la muwerimu d'un maître de takienta. La muwerimu est la "puissance" d'un chef de famille; elle le rend capable de "parler" devant les autels d'ancêtres, au moment d'un sacrifice, par exemple. C'est donc une "capacité de parole", grâce à laquelle est assuré l'entretien des autels dont dépend la continuité de la takienta.

\footnotetext{
is La toilette du mort est considérée corme le travail le plus éprouvant par les deuilleuses désignées pour le faire. Ces fermes appartiennent au clan frère de celui du défunt, les deux clans s'étant scindés dans un passé relativement récent.
} 
Avec son arc, un homme peut également manifester sa tinitidaati, force masculine par excellence, en affrontant la mort à la chasse, à la guerre ou en "enlevant" une femme à un rival' ${ }^{16}$. Un Otammari ne quitte pas son domicile sans suspendre arc et carquois à son épaule gauche, l'arc étant associé à la main (ou côté) gauche considérée comme masculine ${ }^{17}$.

Le jour du décès, l'arc subit un traitement particulier, qui souligne l'identité entre l'arme et son utilisateur défini comme un chasseur et un guerrier. Les femmes ne peuvent pas entonner leurs chants de deuil avant que l'arc du mort n'ait été "renversé" et mis auprès du corps qu'elles veillent sur la terrasse. L'homme habilité à "renverser" l'arc est l'otebita ("celui qui renverse"); c'est le fils préféré du défunt, l'héritier de sa maison et de sa muwerimu. Un arc a une "tête" et un "fondement". Habituellement, un Otammari a toujours soin de tenir le sien "tête en haut". Le jour du décès, l'otebita pose l'arc "tête en bas" sur le sol afin de le débander. Il le "renverse" pour dénouer le lacet qui maintenait tendue la corde en nerf de boeuf et le remplace par un fragment de la corde avec laquelle on attachera le cadavre au brancard. Quand cette opération est terminée, l'un des proches dit: "nous l'avons renversé", signifiant, par ces mots, que le défunt vient de terminer son existence de chasseur. L'arc est alors mis "tête en bas" aux pieds du mort.

- la gibecière de peau de boeuf ou de cabri dans laquelle un Otammari serre pipe et tabac, et, selon ses fonctions ou pouvoirs, cauris et médicaments ${ }^{18}$. Le jour du décès, les ongles et les cheveux coupés sur le cadavre sont mis dans ce sac. La pipe reste posée au dehors, à coté de la calebasse du défunt.

\footnotetext{
16 Le mariage dit "par enlèvement" est le type de mariage secondaire rencontré chez les Batammariba. Plusieurs conflits sanglants entre villages ont eu pour origine, dans le passé, de tels "enlèvements" de femmes dotées.

17 Lă gauche est le côté masculin qui correspond dans la takienta, orientée à l'ouest, au côté sud. Bien entendu, un archer porte toujours, par commodité, son arme au côté gauche. Mais un gaucher de naissance est considéré corme un privilégié et un chasseur supérieur aux autres: à sa mort, on célèbre sa singularité en le nommant le "gaucher". Le statut de l'arc d'un otammari $n$ 'est pas sans évoquer celui de l'arc des Guaraki dont parle P. Clastres dans La société contre l'état (1974).

${ }^{10}$ Ou encore, s'il s'agit d'un devin, son bâton de divination.
} 
- les bracelets (dont on dit qu'ils sont "demandés" par l'ancêtre ${ }^{19}$ ), de fer ou de cuivre, torsadés ou lisses, que, sa vie durant, une personne porte au poignet. Cependant, il est d'usage que contrairement à une femme, un homme garde les siens à son domicile et les remplace par un bracelet unique, représentant la totalité des autres. Le jour du décès, une grande discrétion entoure la manipulation des bracelets. Il est donc difficile de savoir où ils sont déposés au moment où l'on veille le corps. Il faut les distinguer des bracelets, également en métal, simple parure, qui ne sont pas, comme les premiers, la manifestation concrète du "retour" d'un ancêtre dans un individu.

- le couteau, dit "couteau de sacrifice", qu'un Otammari porte suspendu au cou, glissé dans un étui de peau de boeuf retenu par une cordelette, et les flèches à pointes de métal. Le couteau, considéré comme une arme, sera exhibé avec l'arc pendant tout le temps des cérémonies, sans subir de traitement particulier.

Les effets d'une femme comprennent:

- le bâton de marche, kupaati, homologue de l'arc d'un homme. Ce bâton accompagne la femme dans les grandes étapes de son existence. Pour une jeune fille, accepter le kupaati que lui offre un jeune homme est s'engager à l'épouser. Sur ce bâton sont gravées des incisions identiques aux scarifications qui recouvrent le corps d'une femme, après qu'elle ait été initiée au rituel de dikunteri ${ }^{\%}$. Une jeune femme ne peut d'ailleurs exhiber son bâton à la danse qu'une fois initiée elle-même à ce rituel. Le kupaati que brandissent les jeunes épouses durant les fêtes est signe de féminité et de vigueur. physique: avec son bâton, une femme doit savoir repousser un agresseur éventuel lors de ses voyages, ou tuer les serpents qui croisent son chemin. Elle ne s'éloigne pas de sa maison sans cette "arme". Le kupaati lisse, non incisé, est l'apanage des femmes parvenues à la ménopause. Il

\footnotetext{
"Ces bracelets de fer sont très comparables aux bracelets, supports de puissance ancestrale, des Gourmantché (M. Cartry, 1973 et 1987)

20 Ces scarifications se font progressivement, au cours des quatre années précédant dikunteri.
} 
est mis près du corps de la défunte, le jour du décès, et montré publiquement tout au long du rituel funéraire ${ }^{21}$.

- le panier à couvercle, takummanta, homologue du sac de l'homme. Une femme en possède généralement deux. L'un où elle garde ses condiments, l'autre ses parures. C'est le premier, dans lequel on met les cheveux de la morte, qui est exhibé. Le second, pourtant, semble avoir plus d'importance. Dans ce dernier, une épouse range ses biens: vêtements et parures. Quand elle se prépare à quitter son mari pour un autre, elle confie au nouvel élu ce panier, comme gage de sa prochaine venue. Le mari peut surprendre le conciliabule des amants, ou être prévenu par un tiers: il cache alors ce panier, sachant que sa femme ne pourra quitter sa maison si elle ne peut l'emporter. A la mort, il sera objet d'héritage.

- un panier sans anse, servant à transporter farine, graines, haricots... élément primordial d'une "panoplie" féminine.

- un pot à puiser, une calebasse.

- les bracelets "demandés" par l'ancêtre (de sexe féminin, en général). Comme ceux de l'homme, il est difficile de savoir ce qu'il en advient au moment du décès.

\section{Le devenir des ukinenti.}

Les objets métalliques, tant masculins que féminins, sont conservés. Les parures de "beauté" sont objets d'héritage, celles d'un homme reviennent au frère utérin le plus proche en âge, celles d'une femme à l'aînée des filles de la soeur utérine, elle aussi la plus proche en âge. Le couteau et les pointes de flèches d'un homme reviennent également à son frère qui les utilisera. Les bracelets "demandés" par l'ancêtre ne peuvent en aucun cas être détruits. Signe du retour d'un souffle qui peut revenir en d'autres vivants, ils appartiennent de

\footnotetext{
ㄴes femmes initiées appartenant au clan de la défunte dansent auprès de ce bâton à deux reprises: d'abord dans la maison du mari avant que l'on $n^{\prime}$ 'emporte le simulacre dans la maison du père de la morte, puis le jour de la levée de deuil, devant le portail.
} 
droit au fils benjamin, héritier de la takienta (au décès d'un père) ou à la fille aînée de la soeur utérine (au décès d'une mère).

Par contre, les ukinønti périssables sont tous détruits mais à des moments différents.

Le jour de la première levée de deuil, bakunaa, sont brisés puis enfouis sous terre les ukingnti que nous considérons comme des prolongements du corps: gibecière d'un homme, panier à couvercle d'une femme (tous deux contenant les cheveux du défunt), pipe, calebasse des repas, pot à puiser. On dit de ces "effets", enterrés à l'ouest, à la croisée des chemins menant à la maison, qu'ils sont "jetés": on en débarrasse, on en délivre la maison.

C'est bien après la seconde levée de deuil que sera "jeté" d'un homme et le bâton de marche d'une femme. Jusque là, l'arc (attaché au carquois) est gardé dans la division médiane du grenier masculin où l'on a coutume de conserver les biens de valeur, tels que cauris et maintenant argent, et certains objets porteurs d'une force dangereuse. Un jour le frère du défunt, qui en a hérité, décide de s'en débarrasser: "il est temps de le jeter, l'enfant né du souffle de l'ancêtre pourrait le voir". Un Otammari ne doit jamais connaître l'identité de l'ancêtre qui l'a formé: il en mourrait. Apercevoir son arc fait courir le même risque. On le détruira clandestinement. Autant dire que l'arc (ou le bâton de marche) est le double périssable et individualisé des bracelets de métal, qu'il est associé à l'existence de l'être humain marqué par son appartenance sexuelle, un être unique en son genre en lequel le souffle d'un ancêtre est revenu pour un temps limité.

Un geste particulier accompli à la naissance d'un enfant par l'accoucheuse nous invite, également, à considérer l'arc de l'homme ou le bâton de la femme comme l'un des attributs spécifiques donnés par l'ancêtre. Dès qu'elle a coupé le cordon ombilical, l'accoucheuse recueille dans sa main les "affaires" ou tinenti du nouveau-né, invisibles pour tout autre qu'elle-même. Ces tinenti sont logés dans

\footnotetext{
22 Par le terme "jeté", un otamari entend: détruit et enterré. Le mot jeté indique clairement la volonté de se débarrasser des "affaires", en párticulier du tiyoti ("saletés") euphémisme impliquant l'idée d'un danger, d'une force mortifère que recèleraient ces objets.
} 


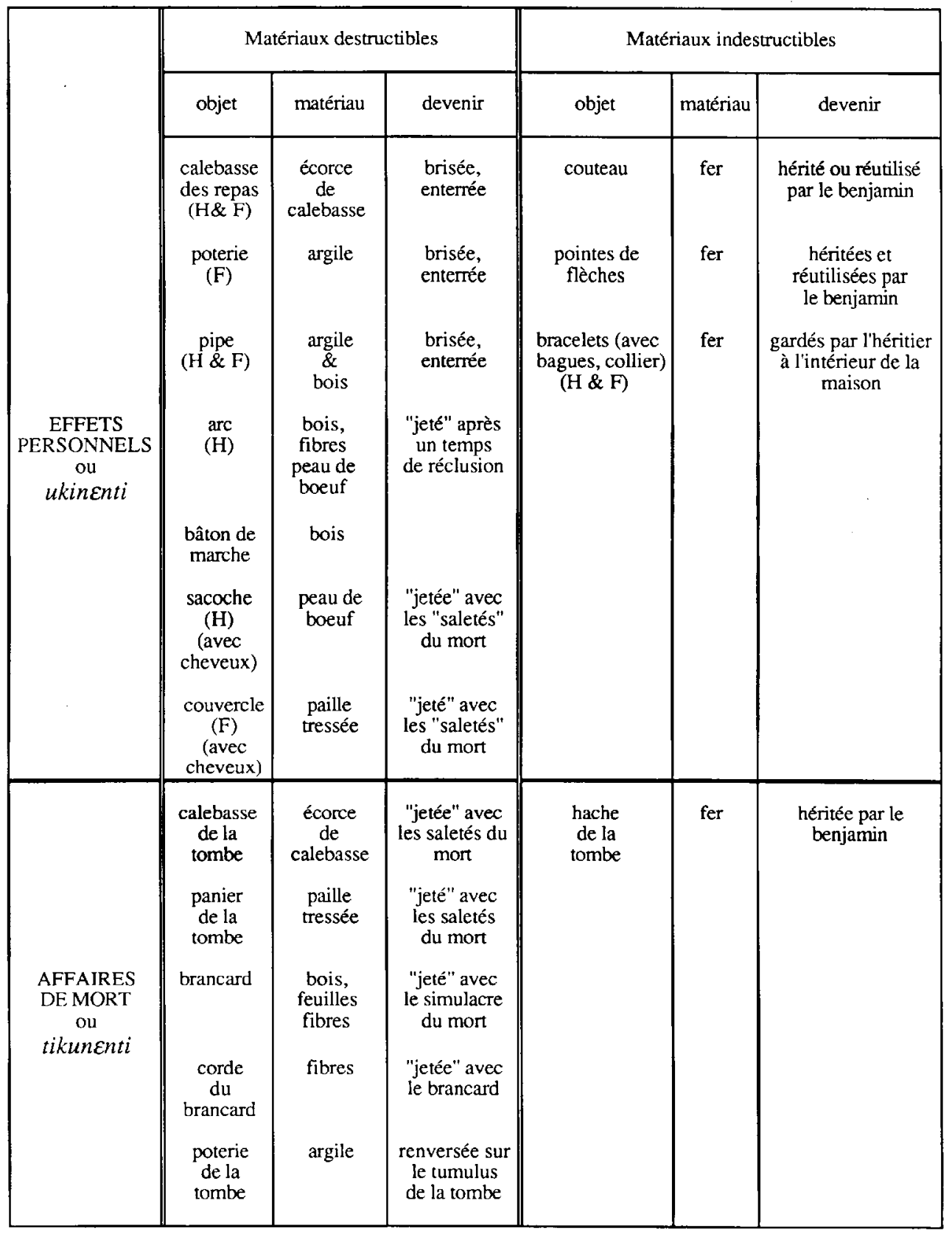

Classement et devenir des tinenti ou "affaires d'un mort" (en dehors des instruments de musique et parures utilisés indifféremment pour tous les défunts d'un même clan ayant droit au rituel des tibenti). $\mathrm{H}=$ homme; $\mathrm{F}=$ Femme . 
les plis du corps de l'enfant; l'ancêtre responsable de la naissance lui en a fait don. Ces dons peuvent être des pouvoirs (de clairvoyance, de divination, de guérison). En principe, un enfant de sexe masculin reçoit toujours, au moins, un arc minuscule et invisible ${ }^{23}$, signe qu'il sera un grand chasseur. Parfois, il reçoit aussi une petite houe, signe qu'il sera un cultivateur renommé. L'accoucheuse remet ces tinenti invisibles au père, qui les roulera dans une motte d'argile, "boule de destin" de l'enfant conservée dans une poterie sur l'autel des ancêtres de la takienta. Dans cette optique, l'arc d'un mort de sexe masculin serait alors cet "effet" invisible à la naissance, parvenu à maturation $\mathrm{du}$ seul fait des initiatives et des actes de courage accomplis durant sa vie par le défunt. Le seul "effet" qui, contrairement aux autres (clairvoyance, divination), ne pourrait se matérialiser que sous l'action d'une volonté et d'un choix.

Que détruit-on alors à travers ces ukinenti "prolongements du corps" brisés et enfouis sous terre le jour de la première levée de deuil ? Se borne-t-on à supprimer les traces d'un corps devenu cadavre, dont l'ombre inquiétante erre encore dans la maison - ainsi que nous l'avons souvent entendu dire ? Cette destruction paraît inséparable d'un réajustement des rapports entre le défunt et les survivants. C'est du moins ce que semble suggérer un rite auquel nous avons assisté quelques jours avant la levée de deuil d'une mère. Les trois jours qui succèdent à l'enterrement d'un ancien sont employés à préparer la bière de mil dont on arrosera la tombe le quatrième jour, le jour du bakunaa. Pendant ces préparatifs, le conjoint quel que soit son sexe, doit rester reclus. Mais cette réclusion est ponctuée par une série de repas ritualisés qui, peu à peu, favorise un retour à la vie "normale". Chacun de ces repas, en effet, vise à instaurer un nouveau type de relations entre le conjoint survivant et le défunt, ce conjoint étant successivement considéré comme un époux et comme un père (ou une mère). Deux femmes jouent un rôle prépondérant dans la mise en place de ces nouveaux liens. L'une appartient au clan

${ }^{23}$ Sur le statut mythique de I'arc, voir P. Jespers, 1983. 
du veuf (ou de la veuve): elle représente la fille. L'autre, appartenant au clan du défunt, représente l'épouse (ou l'époux).

Le réajustement des nouveaux rapports est entériné le jour du bakunaa par la destruction des effets personnels du mort. Précisons encore que le rite se déroule de façon identique quel que soit le sexe du défunt.

\section{Renouer avec la défunte, détruire ses "affaires"}

Une mère vient d'être enterrée. En raison de son âge, elle a eu droit au rituel des tibenti: on lui a creusé une nouvelle tombe. Jusqu'au jour du bakunaa, le veuf ne peut aller dans d'autres majsons. Il ne peut pas boire ou manger en compagnie de ses enfants: "la mort de son épouse est sur sa tête" (plus précisément sur ses cheveux). Il reste dans sa case personnelle, attenante à la takienta familiale. Par l'ouverture, on l'entrevoit accroupi, le front baissé, la peau couverte de poussière. Il ne parle pas. Trois jours avant le bakunaa doivent commencer les préparatifs de la bière de mil que les oncles utérins de la défunte boiront sur la tombe de celle-ci, au cimetière du clan de son père. Le lendemain du tibenti, le responsable des cérémonies se rend à la maison du veuf. Assis près du portail, il assiste à l'arrivée des filles mariées, soeurs de kunalakua, venues prendre le mil que l'on a mis à germer sur la terrasse depuis deux jours. Elles l'écraseront dans la maison de leur mari.

\section{Le premier jour}

Le matin, le veuf est dans sa case. Les filles mariées rapportent le mil écrasé et disposent deux jarres de grande dimension sur deux tranchées creusées en croix où brûle un feu. Pour la première fois depuis des semaines, la cour de la maison s'emplit de rires. L'animation des jeunes filles tire la maison du froid où l'avaient figée la longue maladie puis la mort de la mère. 
Dans la soirée, le veuf sort de sa case pour la première fois et s'adosse à la facade. Une femme âgée ${ }^{24}$ lui coupe les cheveux à ras. Elle fait de même avec chacun de ses enfants et petits-enfants des deux sexes. Le responsable des cérémonies dépose près du veuf les tinenti de la morte que sont le panier à couvercle, le pot à puiser, le panier sans anse contenant calebasse et bâton de marche, la pipe, le balai de chaume. A coté, sont posées les "saletés" de la morte. Tous ces objets sont exposés sur le coté nord et féminin de la maison, au dessous du grenier de l'épouse.

Avant le crépuscule, devant la maison, l'animation est à son comble. Personne ne semble se soucier du veuf. Des fillettes vont et viennent remplissant des jarres d'eau. D'autres préparent le repas du soir. Le veuf, la femme qui vient de lui raser les cheveux et deux autres femmes âgées s'installent dans la cour de la petite case. Le muret qui entoure la case les protège des regards. Une calebasse contenant une boule de pâte de mil est posée sur le sol. Le veuf est en face des trois femmes assises. L'une des nouvelles venues fait partie du kunalakua de son épouse, elle la représente: nous la nommerons l'épouse. L'autre femme tient des fonctions rituelles à l'intérieur du propre clan du veuf; elle tient, ici, le rôle de la fille benjamine: nous la nommerons la benjamine. Tout d'abord, l'épouse, qui a préparé la pâte de mil, met une bouchée dans la main gauche du veuf: il l'avale, puis une deuxième, qu'il avale également. Il continue à manger en se servant de sa main droite, en compagnie de l'épouse qui partage son repas. Les deux autres femmes se contentent de les regarder. Ayant fini de manger avant l'épouse, le veuf demande: "je peux sortir ?". "Attends-moi" répond-elle; elle termine son repas et se lève. Ils sortent ensemble et vont rejoindre le cercle familial devant la maison. Aucune des personnes s'affairant là ne tourne la tête vers le baobab sous lequel viennent de s'asseoir le veuf et l'épouse, comme un couple. Ils devisent amicalement. L'épouse fait pivoter son fuseau en s'entretenant avec le veuf qui rompt pour la première fois son mutisme.

\footnotetext{
24 Elle enlève ainsi l'empreinte de l'ombre (uffenku) de la morte. Cette fenme est chargée de laver et de oindre les cadavres dans le clan de la défunte.
} 
A la tombée de la nuit, le veuf regagne sa case qu'il ne quittera plus jusqu'au matin. Pendant toute la nuit, il y aura une grande animation autour des jarres dans lesquelles la bière bout à gros bouillons.

Selon un jeune père de famille âgé d'une trentaine d'années, l'épouse, à ce premier repas, a mis les bouchées de pâte de mil dans la main gauche $d u$ veuf parce qu'il s'agit "du dernier repas donné par l'épouse à son mari". La main gauche au lieu de la droite normalement utilisée parce que l'épouse est morte. L'une des bouchées est destinée à la défunte, l'autre est pour l'époux. Le choix de la main gauche indique la séparation du mari d'avec sa femme et sa solitude. Les deux bouchées nettoient les "saletés" de l'épouse qui souille la tête du veuf.

Un père de famille plus âgé (une cinquantaine d'années) donne de ce repas une interprétation légèrement différente. Selon lui, l'épouse met les bouchées dans la main gauche du veuf pour lui montrer que "si sa femme était encore en vie, il ne mangerait pas de cette main. C'est comme si l'on donnait à manger à un enfant. Il se montrera maladroit, avalera avec difficulté et les vieilles lui diront: "c'est toi qui a mangé ta femme (tu es un sorcier) !" Sa maladresse préfigure les peines qui seront désormais son lot.

Il est un fait que l'épouse, ici, n'entend ni supplanter l'épouse défunte, ni l'imiter. Elle jouerait plutôt le rôle de la "mère" du jeune initié qui, lors du difuani le nourrit pour la première fois après une semi-réclusion de trois jours - bien qu'à difuani le rôle de la mère soit tenu par un parrain nommé "mère". On peut également supposer que l'épouse personnifie l'ombre, kufenku, de la défunte, rôdant encore autour de la takienta et exerçant son emprise sur son mari. $\mathrm{Au}$ cours de ce repas, les positions respectives du veuf et de son épouse défunte se trouvent clairement définies. Ce soir-là l'ombre de la morte se détache du veuf pour investir l'épouse qui initie le mari à son veuvage et à sa solitude.

\section{Le deuxième jour}

Le lendemain, tous les membres du kunalakua auquel appartient le veuf ont envahi le devant de la takienta. Les jarres fument, les rires fusent. Nul ne songe à s'intéresser à la scène muette qui se 
déroule discrètement de l'autre coté du muret, où vers neuf heures, le veuf, l'épouse et la benjamine se sont à nouveau installés, dans la cour de la petite case. Comme la veille, c'est l'épouse qui a préparé le repas composé d'une calebasse de pâte de mil et d'un bol de sauce. Le veuf s'asseoit en direction du nord, sur un petit tabouret, face à la benjamine accroupie devant lui. Celle-ci détache une bouchée de pâte, la trempe dans la sauce et la jette sur le coté gauche du veuf. Puis elle se rince les doigts à l'eau d'une calebasse, se lève et s'en va. L'épouse se met à son tour en face du veuf, détache avec sa main droite une bouchée, l'élève à la hauteur des lèvres de celui-ci et la rejette sur sa gauche (à lui). Elle fait de même avec une autre bouchée, qu'elle rejette sur la droite. Ensuite, elle pose successivement deux bouchées sur le dos de la main gauche du veuf qu'il rejette une fois à gauche, une fois à droite.

Le veuf change alors de place et s'asseoit en direction de l'ouest. Accroupie en face de lui, l'épouse met successivement dans sa main gauche deux bouchées qu'il avale. Ensuite, il prend lui-même, de la main gauche, deux nouvelles bouchées puis continue à manger en se servant de la main droite. L'épouse partage son repas.

Selon l'informateur âgé de cinquante ans déjà cité, la benjamine aurait jeté une bouchée à gauche et à droite du veuf pour que celui-ci prenne conscience de l'absence de son épouse: "c'est elle, normalement, qui aurait dû lui présenter la nourriture... Quant à l'épouse, elle lui a montré, en lui donnant à manger, qu'elle avait pitié de son état. Mais elle a mis la pâte dans sa main gauche pour lui signifier les souffrances qui l'attendent désormais".

Les explications données par un Otammari habitant le Bénin complètent cette interprétation: "le veuf s'asseoit d'abord en direction du nord pour s'identifier aux premiers ancêtres des Batammariba, venus jadis de Dinabaa (situé au nord). Il regarde ensuite vers l'ouest, qui est la direction prise par les défunts, pour se tourner vers son épouse, en route vers le village des morts. En nourrissant le veuf, l'épouse qui appartient au kunalakua de la défunte, montre qu'elle maintient l'alliance entre les deux familles."

Ainsi, au cours de ce deuxième repas, l'épouse intervient en tant qu'alliée de la famille du veuf, soucieuse de maintenir les liens entre 
les deux takienta. La benjamine, quant à elle, ne nourrit pas son père: elle ne saurait devenir une épouse. A qui sont destinées les bouchées successivement jetées à droite et à gauche du veuf ? En l'état actuel de nos recherches, nous nous bornerons à signaler l'identité de ce geste avec celui qui confirme le passage à l'âge adulte des jeunes gens à la fin de l'initiation, et avec celui qui clôture la construction de l'autel de l'ancêtre "revenu" dans un nouveau-né.

Dans la soirée, le veuf, la benjamine et l'épouse s'installent à nouveau dans la cour de la petite case pour le repas du soir. Il fait encore jour ${ }^{25}$. L'épouse a préparé le repas. Le veuf s'asseoit en direction du nord. L'épouse dépose à la droite de celui-ci une calebasse contenant un mélange de pâte de mil et de fonio et, à sa gauche, un bol de sauce où trempe un morceau de boeuf. La benjamine détache une bouchée, la jette à la gauche du veuf, puis une autre qu'elle jette à la droite ${ }^{25}$. Ensuite, elle élève une bouchée à hauteur des lèvres du veuf pour la rejeter entre les jambes de son "père". Enfin, par deux fois, elle pose sur le dos de la main gauche du veuf une bouchée qu'il rejette une fois sur la gauche, une fois sur la droite. Il détache alors deux bouchées avec sa main gauche, les avale et continue à manger avec la main droite, "normalement". L'épouse observe attentivement toute la scène.

Selon l'informateur venant du Bénin, les gestes accomplis par la benjamine "indiqueraient que, désormais seul, le veuf peut néanmoins compter sur la sollicitude de sa fille benjamine, bien que le rôle de cette dernière ne puisse jamais s'assimiler à celui d'une épouse".

De fait, les repas sont, ici, toujours préparés par l'épouse, et la benjamine ne nourrit jamais son "père". Mais, en jetant une bouchée en direction du sexe du veuf, la benjamine semblerait confirmer l'appartenance d'une fille au kunalakua et à la takienta de son père. En effet, son geste évoque celui que fait le devin avec son bâton quand un consultant souhaite identifier un ancêtre. Le devin pointe son bäton vers le sexe du consultant pour indiquer que cet ancêtre appartient à la lignée paternelle.

\footnotetext{
25 Jusqu'à la première levée de deuil, il est interdit au veuf de manger après la tombée de la nuit car les ombres des mauvais morts errent à la recherche de vivants pour s'en saisir.

${ }^{26}$ Peut-être offre-t-elle de la nourriture à l'ancêtre "revenu" dans le veuf?
} 
En résumé, ce repas réaffirme les liens du veuf avec ses filles représentées par la benjamine - mais des liens mettant en évidence moins la disparition de la mère, que la "mise à distance" de celle-ci: l'épouse est présente sans intervenir comme sera présent l'autel de la défunte qui, après la seconde levée de deuil, sera élevé dans la takienta.

\section{Le troisième jour, jour de bakunaa}

Avant l'aube, les tinenti personnels et périssables de la défunte sont brisés et enfouis sous terre à la croisée des chemins menant à la takienta. Avant l'aube, également, les parents utérins de la défunte boivent la première bière, encore brûlante. Ils goûtent le première pâte de mil qu'ils consommeront plus tard au cimetière et à la takienta du père de la défunte. Seuls sont servis les fils et les filles ainés. $\mathrm{Au}$ matin, tous les membres du clan auquel appartient le kunalakua du veuf envahissent la cour. Chaque maison a fait apporter plusieurs pots de bière, l'animation est grande. Dans la petite cour, le veuf et l'épouse s'asseoient devant le repas que cette dernière a préparé. L'épouse dit au veuf: "aujourd'hui tu manges seul et "normalement" (avec la main droite)". Ils partagent le repas.

Dans la soirée, les oncles utérins de la défunte accompagnés des frères et des fils aînés se rendent au cimetière. Deux femmes âgées tenant le rôle de fille aînée dament la terre devant la poterie fixée sur le tertre funéraire. La poterie représente la nouvelle maison de la morte, le devant damé, la terrasse. Avant qu'elles n'effectuent cette opération, la houe et le pieu des fossoyeurs, la kuya, ont été retirés du tertre où on les avait plantés après l'inhumation. Une fois la tombe définitivement "fermée", une bouillie de mil est versée sur la poterie, suivie de bière de mil que se partagent ensuite les participants. La cérémonie terminée, tous retournent à la takienta. Quand ils sont en vue de la maison, le veuf rentre dans sa case en compagnie de l'épouse qui lui a préparé un dernier repas. Il mange "normalement" avec sa 


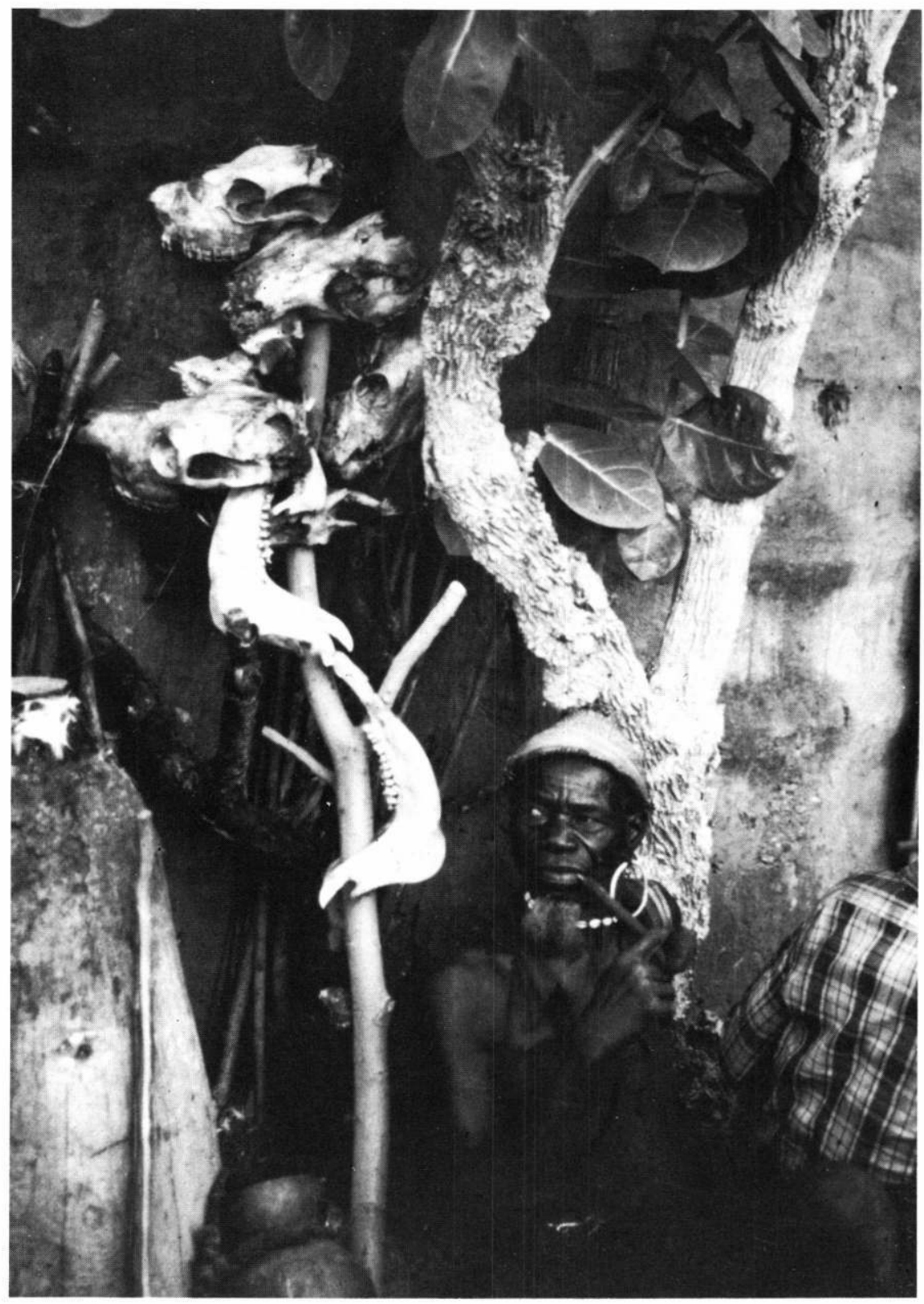

tiyoti: les "saletés" du mort (cliché M. Smadja) 


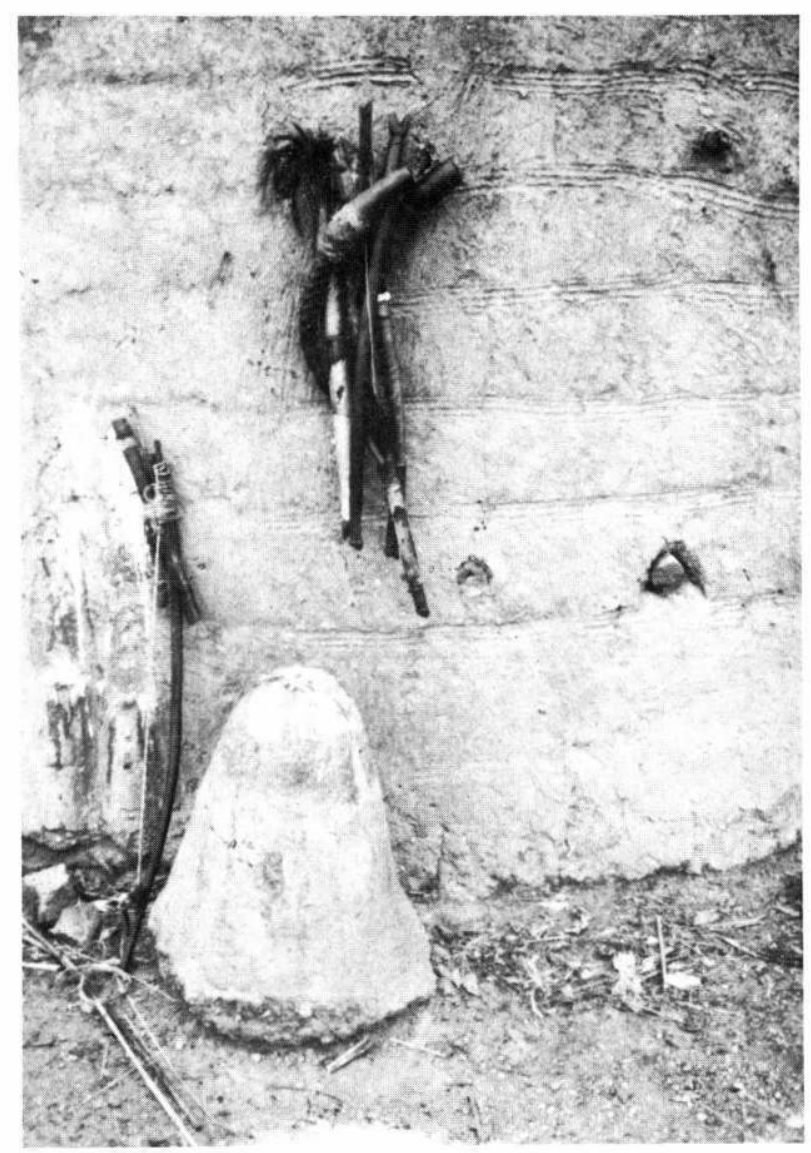

Les affaires du mort accrochées au-dessus d'un autel de terre (Cl. M. Smadja)
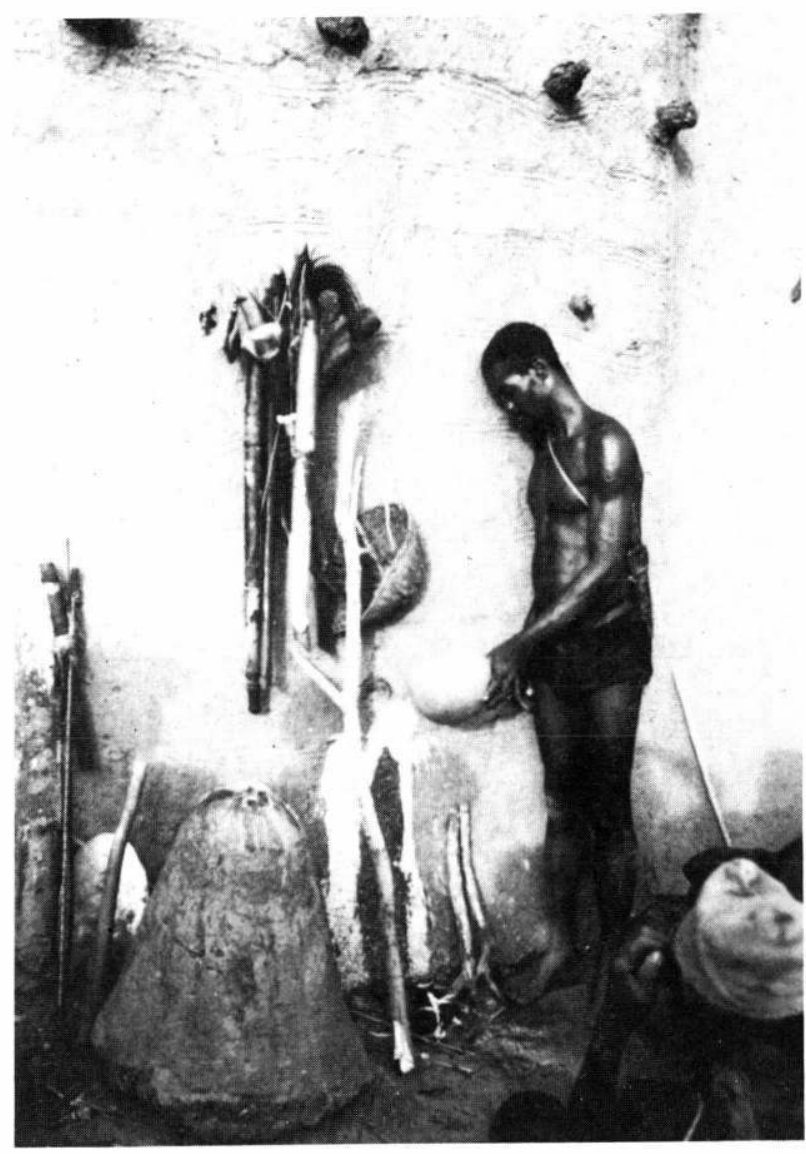

.

Libation de bière de mil sur les "affaires du mort" le jour de la première levée de deuil ( $\mathrm{Cl}$. M. Smadja) 


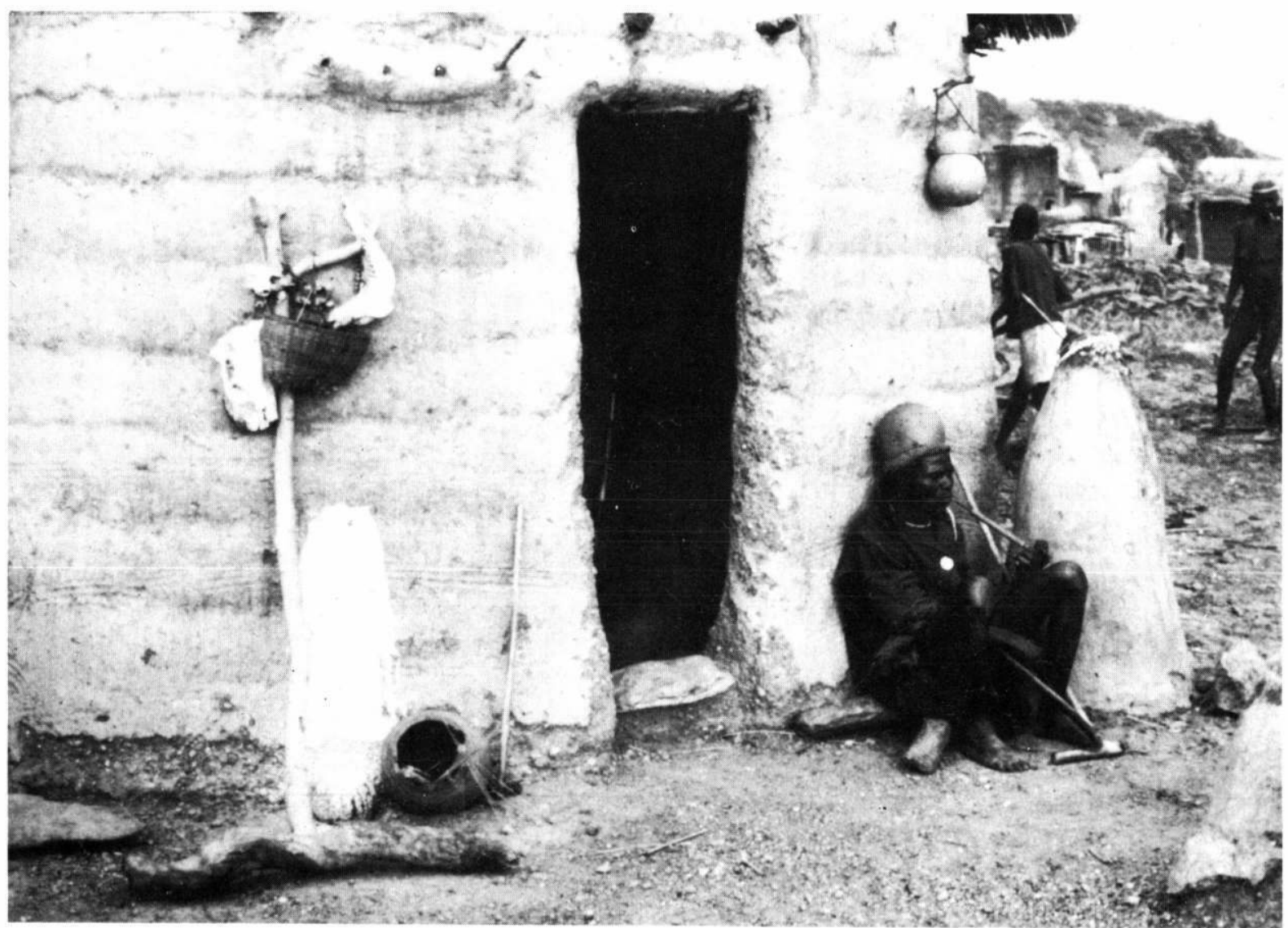

tiyoti: les "saletés" d'une défunte ( $\mathrm{Cl}$. M. Smadja) 


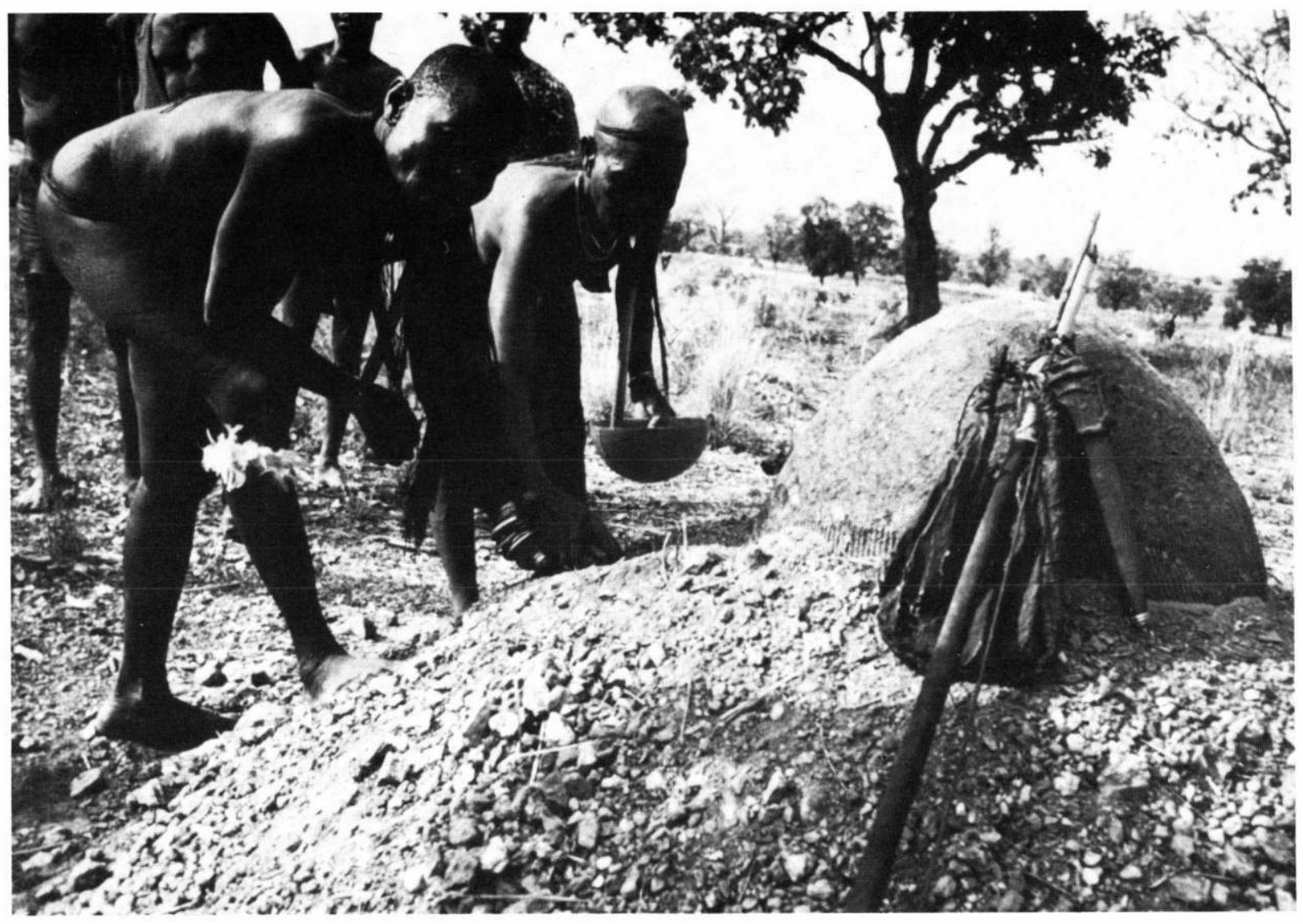

Libation de bière de mil sur la tombe le jour de bakunaa Au premier plan: les "affaires" du mort ( Cl. M. Smadja) 
main droite, partageant la pâte avec sa compagne, ne semblant nullement tenir compte du retour de la procession ${ }^{27}$.

Si le veuf mange normalement le matin de la levée de deuil, c'est qu'un peu avant l'aube les effets personnels et périssables de son épouse ont été, selon la terminologie des Batammariba, "jetés". Le retour à la vie normale du veuf coïncide donc avec la destruction des "affaires" liés au corps vivant de son épouse. Il s'effectue aussi au moment où la "bière de mort" est prête. Versée sur la tombe, cette bière parachèvera la fermeture de la sépulture, assimilée au dernier acte de construction de la demeure où est maintenant installée la défunte. Celle-ci ne rôdera plus autour de la maison sous forme de spectre ou d'ombre, elle ne s'agrippera plus aux cheveux de son époux ou de ses enfants. La destruction des effets "prolongements du corps vivant" confirme bien l'évacuation de l'ombre, cette opération ne pouvant se faire qu'après l'instauration d'un nouveau type de relations entre les survivants et le mort, en particulier, ici, entre le veuf et son épouse. En ce sens, on peut dire que les tinenti sont détruits quand il n'y a plus de raison de maintenir les liens qu'ils représentent sous leur ancienne forme. La femme qui joue l'épouse au cours des repas a peu à peu abandonné ce rôle pour ne plus jouer que le sien: celui d'une belle-soeur amicale, soucieuse de maintenir l'alliance entre les deux maisons.

Cependant, un dernier rite, qui a lieu le lendemain de la levée de deuil, semble inviter la défunte à se souvenir du chemin de la takienta de son mari comme de celle de son père: à y retourner, non plus sous forme d'ombre ou de spectre, mais comme diyanni - ce souffle qui doit revenir dans un nouveau-né. Le rite circonscrit le lieu où devra se fixer cette puissance: à proximité et à l'entrée de chacune des takienta.

Le jour qui suit la levée de deuil

Le matin, près du portail, sur le coté nord et féminin de la maison du veuf, une pintade est sacrifiée sur un débris de marmite ayant

\footnotetext{
$27 \mathrm{~S}^{\prime}$ il s'agit d'une veuve et non d'un veuf, ces repas rituels se déroulent de la même manière. Le rôle du défunt est tenu par une femme appartenant au cían du mari.
} 
appartenu à la défunte. Le débris, hérissé de duvets, est posé à l'endroit où l'on a enterré les tincnti. Trois femmes appartenant au sous-clan complémentaire de celui du veuf s'accroupissent en triangle près du débris. L'une regarde vers l'ouest, les deux autres, en vis-à-vis, sont placées respectivement au nord et au sud. Le fils aîné reste debout. Les deux femmes en vis-à-vis tiennent ensemble deux feuilles de karité tête bêche, la partie brillante sur le dessus. La troisième femme $\mathrm{y}$ dépose la "nourriture de la morte": une toute petite quantité d'un mélange de pâte de mil et de fonio, un filament de poisson blane "du marigot", un peu de sauce aux graines de baobab, le tout arrosé de bière de mil. Le fils aîné ajoute un brin de tabac. Puis ils attendent tous quatre dans un silence absolu, sans bouger. Enfin, une mouche attirée par l'odeur, voltige au dessus du "plat", repart, hésite, revient ... et se pose sur les aliments. En un éclair, les deux femmes enroulent les aliments, tenant prisonnière la mouche qui représente la défunte. Le rouleau est dissimulé dans une touffe d'herbe, à l'endroit où quelques semaines plus tard, sera élevé un petit autel de terre, relais entre la tombe et la takienta.

Puis les femmes s'installent de part et d'autre du portail, accompagnées du fils aîné, et recommencent la même opération avec deux autres feuilles. Cette fois le rouleau est caché près des autels des fondateurs de la,takienta. Nommés Soleil et Terre, ces autels sont plaqués sur le côté sud, à la base de la façade.

Les jeunes filles balayent alors l'intérieur de la maison ${ }^{2 *}$, et hommes et femmes du clan du mari se rendent à la maison du père de la défunte où une cérémonie identique se répète, cette fois conduite par deux femmes appartenant au sous-clan complémentaire de celui de l'épouse, mais en présence de l'une des trois premières.

Quand, après avoir attrapé une mouche à la croisée des chemins, elles s'accroupissent devant le portail, l'impatience les gagne. La chaleur est intense et la mouche tarde à faire son apparition. "Rentre, on veut s'en aller !" s'écrie l'une. Et l'homme qui vient de sacrifier la pintade, sur un ton de colère: "mais que fait-elle ? Qu'elle se dépèche : On en a assez d'attendre !" Après avoir enfin roulé une

\footnotetext{
28 Le balayage est destiné à débarrasser la maison des derniers vestiges dangeureux de la morte.
} 
mouche dans les feuilles et caché le paquet près des autels de l'entrée, les femmes se partagent, dans le vestibule, la "nourriture de la morte" composée des mêmes aliments qui ont attiré la défunte. Nourriture particulièrement dangereuse pour un homme et qu'elles seules peuvent impunément consommer à condition de rester dans le lieu qui sépare le dedans du dehors de la maison. Le repas terminé, les femmes appartenant au clan de la défunte, et peut-être initiées en même temps qu'elle au temps de leur jeunesse, forment un grand cercle et, comme les jeunes initiées rassemblées devant la maison de l'une d'entre elles le dernier jour de la cérémonie, entonnent le chant qui clôture dikunteri.

Quelques mois plus tard, après la seconde levée de deuil, la takienta du veuf comme celle du père possèderont un autel de terre, réceptacle du diyanni de la défunte et double de sa tombe. Le diyanni pourra ainsi, en toute liberté, circuler de la tombe à la takienta .

Il faut longtemps pour qu'un mort apprenne à mourir et ne désire plus rien de sa vie antérieure. Quand, une à une, les affaires qui le retenaient en arrière auront été détruites, quand il sera devenu un souffle sans mémoire, il pourra désirer être à l'origine d'une nouvelle vie.

Les seules traces que garderont de lui les vivants seront des dessins gravés sur des bracelets de métal et un nom que l'on dira "dans son ventre", sans plus jamais le prononcer à haute voix. 


\section{Références bibliographiques}

Cartry, M.

1973 "Le lien à la mère et la notion de destin individuel chez les Gourmantché", in La notion de personne en Afrique Noire, Paris, CNRS, 255-282

1987 "Le suaire du chef", in Sous le masque de l'animal. Essais sur le sacrifice en Afrique noire, M. Cartry éd. Paris, PUF, $131-231$

Hertz, R.

1928 "Contribution à une étude sur la représentation collective de la mort", in Mélanges de Sociologie Religieuse et Folklore, Paris, Alcan (1ère ed. 1907).

Jespers, $\mathrm{Ph}$.

1983 "L'arc et le sang des chiens", Systèmes de Pensée en Afrique Noire, 6, Paris, EPHE-CNRS, 65-102

Mercier, Paul

1968 Tradition, changement, histoire. Les Somba du Dahomey septentrional, Paris, Anthropos. 